\title{
3D Anatomy of the Developing Heart: Understanding Ventricular Septation
}

\author{
Timothy J. Mohun ${ }^{1}$ and Robert H. Anderson ${ }^{2}$ \\ ${ }^{1}$ The Francis Crick Institute, London NW1 1AT, United Kingdom \\ ${ }^{2}$ Cardiovascular Research Centre, Institute of Genetic Medicine, Newcastle University, Newcastle upon Tyne \\ NE1 7RU, United Kingdom \\ Correspondence: tim.mohun@crick.ac.uk; sejjran@ucl.ac.uk
}

Understanding how the four-chambered mammalian heart is formed from a simple, looped tube remains challenging, notwithstanding the descriptive accounts left by generations of cardiac anatomists. Much of the difficulty lies in attempting to visualize an intricate series of morphological transformations through the restrictive lens of two-dimensional images derived from histology. Modern imaging methods offer a way to overcome this limitation by providing comprehensive and high-resolution image sets of the developing heart. We have used one such method, high-resolution episcopic microscopy (HREM), to obtain virtual three-dimensional (3D) models of successive stages in mouse heart development. Taking advantage of the ability afforded by 3D modeling to view each heart in any orientation or erosion plane, we provide an illustrated account of how the mouse heart divides into left and right ventricular chambers, and how each acquires its own distinct outflow vessel.

$\mathrm{O}^{\mathrm{f}}$ all the many extraordinary changes that occur during development of the mammalian heart, perhaps the most challenging to understand is the transformation that establishes separate left and right ventricular chambers, each with its own distinct arterial root and vessel. Because the heart initially forms as a simple, looped contractile tube, its conversion to the mature chambered structure requires several distinct and coordinated changes. One of these is the apparently simple division of the ventricular loop into left and right chambers via the growth of an interventricular muscular septum. Expansion of the atrioventricular canal ensures that both of the forming ventricles have an inlet to receive blood from the appropriate atrium. The final change is the division of a single common outflow originating from the original looped heart tube into the intrapericardial aorta and pulmonary trunk. This provides each chamber with its own arterial outflow and enables separation of the systemic and pulmonary circulations.

Understanding how these changes are achieved has proved enormously challenging, and not just because of their morphological complexity. The difficulty is also the consequence of a simple and obvious topological observation. Subsequent to expansion of the atrioventricular canal, the common outflow lies asymmetrically in the early heart, sitting

Editors: Benoit G. Bruneau and Paul R. Riley

Additional Perspectives on Heart Development and Disease available at www.cshperspectives.org

Copyright (C) 2020 Cold Spring Harbor Laboratory Press; all rights reserved; doi: 10.1101/cshperspect.a037465

Cite this article as Cold Spring Harb Perspect Biol 2020;12:a037465 
above, and sharing a largely common lineage with, the future right ventricle (Fig. 1).

With such an arrangement, how then can the left and right ventricles be separated from each other without confining both arterial roots exclusively to the right chamber? Answering this question not only reveals the exquisite choreography of morphological change that underpins separation of the chambers. It also sheds light on an apparently diverse range of congenital heart defects, ranging from malformation or aberrant positioning of the arterial roots to the multiple defects, which result in abnormal retention of interventricular communication even after chamber formation.

Cardiac anatomists have long grappled with the issue of how the left and right sides of the heart are separated. It is testament to their skill and prescience that they have given us such descriptive accounts, despite being restricted almost exclusively to analysis of histological studies. Although their efforts have provided a remarkably accurate outline for many facets of cardiac development, in our view the various accounts addressing ventricular septation and formation of the arterial roots remain the least compelling and most opaque of their legacies.

In recent decades, such anatomical issues have often been eclipsed by the avalanche of advances in genetic and molecular studies of cardiac development. But those same advances have increasingly demanded a morphological framework with which to provide a context for understanding their significance, bringing a renewed relevance to descriptive cardiac anatomy. Parallel advances in imaging technology have also freed us from the constraints that hampered previous generations. We can now combine a variety of imaging modalities with computerbased three-dimensional (3D) reconstruction to interrogate the topology of the developing heart in remarkable detail.

Here we reexamine the manner in which separate ventricular chambers and their arterial roots are established during mouse cardiac development. Our studies use a library of novel image data obtained from high-resolution episcopic microscopy (HREM) of mouse embryo hearts. Our efforts, however, also rest heavily upon foundations provided by previous generations of cardiac anatomists. Their work is now widely accessible, either from primary literature or summarized in secondary reviews and textbooks. For this reason, we have eschewed referencing their individual contributions. Instead, we have revisited the events surrounding ventricular septation, using 3D modeling to provide a fresh perspective on the transformations in heart structure. We hope that our approach illustrates and clarifies the sequence of morphological changes that underpin this critical step in cardiac development.

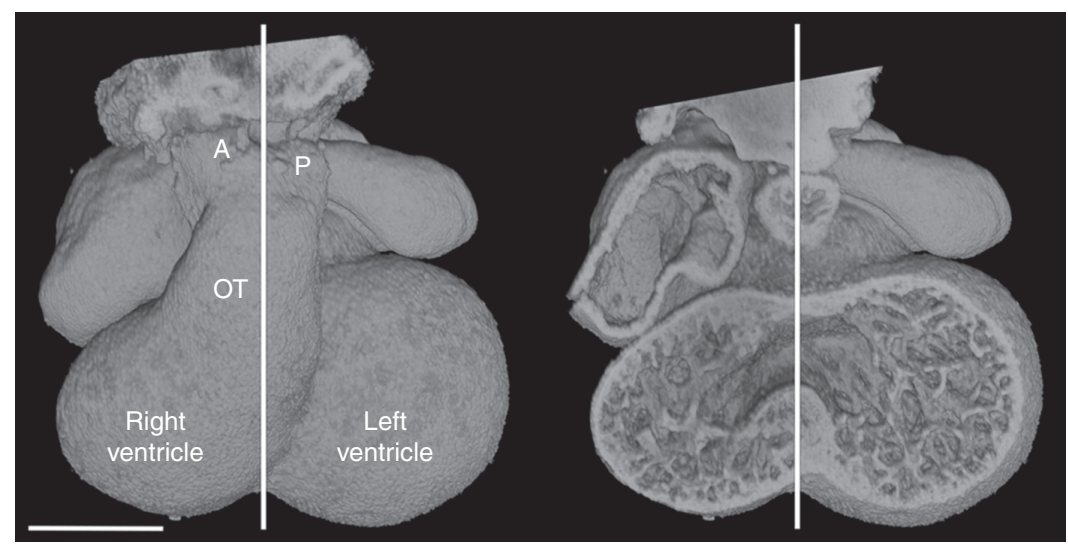

Figure 1. E11.5 heart, with midseptal plane marked. The distalmost end of the outflow tract (OT) divides into distinct pulmonary (P) and aortic (A) vessels lying on either side of the midseptal plane. Scale bar, $0.5 \mathrm{~mm}$. 


\section{HOW FAR DOES THE OUTFLOW TRACT, AND THE VESSELS TO WHICH IT GIVES RISE, CHANGE POSITION DURING CARDIAC DEVELOPMENT?}

In the mouse, division of the common outflow tract occurs during the 1-day window of E11.5E12.5. Because the base of the outflow tract lies over the right ventricle at E11.5, the pulmonary trunk, as it is separated from the aorta, is already positioned appropriately to drain its ventricle. In contrast, without change in either location or orientation, the newly formed aortic root would occupy a comparable but inappropriate position, and the left ventricle would lack any direct outlet. To resolve this topological conundrum, it has been suggested that the outflow vessel shifts and rotates to a more midline position between the left and right sides of the heart, so-called "wedging." It is then further suggested that, to establish a connection with the left ventricle, the aortic root shifts its position leftward.

Inspection of 3D models covering this period provides little support for this view. When newly formed, the roots of both the pulmonary trunk and aorta retain their position lying above and to the right of a plane bisecting the muscular ventricular septum (Fig. 2).

Septation of the outflow tract itself proceeds in a distal to proximal direction, with the result that, although distinct pulmonary and aortic trunks first replace the distal portion of the outflow, the medial region remains common, encompassing the newly appearing primordia of the arterial roots. These latter components are readily recognized by their characteristic trifoliate structure. Forming within a common vessel, and by a shared process, these primordia necessarily lie on the same plane (Fig. 3).

As outflow septation proceeds, the two nascent valves become separated by arterial walls in the pulmonary and aortic roots, and immediately diverge in their individual planes. The pulmonary root retains a similar axis to that of the original outflow tract. The aortic root, in contrast, shifts its axis such that the plane of the valve comes to provide a mirror image of its pulmonary counterpart (Fig. 3).

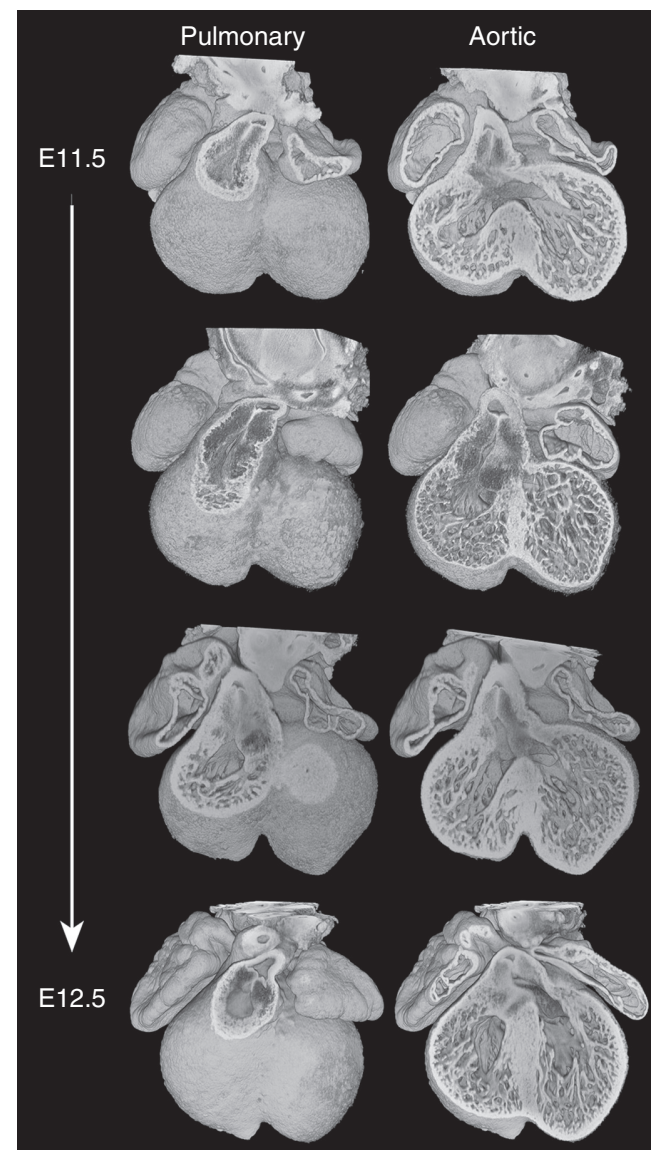

Figure 2. Models of hearts at four successive stages between E11.5 (top) and E12.5 (bottom), eroded to approximate the midvalve level for pulmonary and aortic valves.

Both valve primordia now lie slightly closer to the midseptal plane, presumably as a result of growth and development of the supporting collar of ventricular tissue. The pulmonary valvar primordium now variably straddles the midseptal plane, whereas the aortic valve remains somewhat further to the right, despite its change in plane. This arrangement of the roots is largely unchanged during the next 2 days of development, during which septation of the left and right ventricles is completed and each chamber acquires its own outflow tract (Fig. 4).

In the remaining 4 days of development, from E14.5 to E18.5, both arterial valves do shift leftward. At E16.5, the pulmonary valve lies for the first time on the left, whereas the aortic valve 


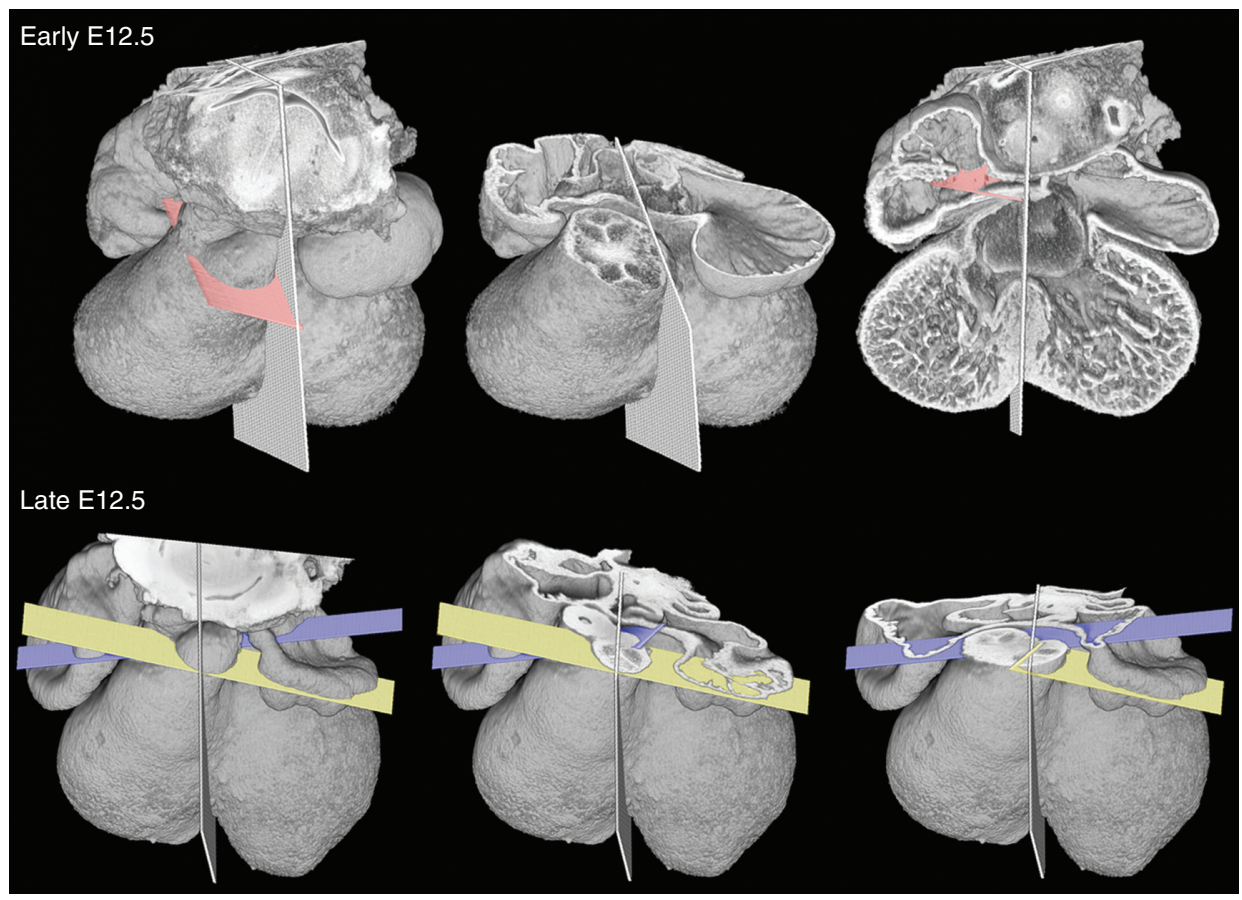

Figure 3. Early and late E12.5 hearts, with midseptal plane indicated. At the earlier stage, separation of aortic and pulmonary roots only extends through the distal outflow tract. Within the medial portion, the primordia of the aortic and pulmonary valves lie on a common transverse plane (pink) through the outflow. By late E12.5, separation of the aortic and pulmonary roots extends through this region. The two vessels adopt divergent orientations, as revealed by the symmetrical arrangement of their valve planes (yellow and mauve, respectively).

straddles the midseptal plane. By full term, at E18.5, both valves lie immediately to the left of the midseptal plane and are positioned over the left ventricle, although the pulmonary valve retains its origin from the right ventricle (Fig. 5).

In summary, for the entire period during which the left and right ventricles become functionally separated (E11.5-E14.5), their developing outflow tracts remain asymmetrically positioned on the right of a plane separating the two ventricles. No major leftward shift of the aortic root can be seen. Such a shift occurs only in the few days before full term. Instead, the most obvious change is a shift in the axis of the aortic root and the corresponding plane of the aortic valve. This occurs immediately after the valvar primordia become separated by ingrowth of their own distinct walls. As a result, how the left ventricle acquires its connection to the aortic root without ongoing interventricular communication remains a topological puzzle.
Its solution lies in other changes to heart morphology during this period, in particular the role played by the cushions of the outflow tract.

\section{ARCHITECTURE OF THE COMMON OUTFLOW TRACT}

Between E10.5 and E11.5, the outflow tract draining the ventricular loop acquires two major cushions, which run along much of its length. Their arrangement establishes the relative positions of the pulmonary and aortic channels even before their separation into the pulmonary and aortic trunks.

At E10.5, the two outflow cushions appear at first in an apparently rather uniform manner, arranged concentrically around the central and proximal portions of the outflow tract. As their distinct and abutting edges become clear, it is evident that the cushions are already in a spiral arrangement, rotating clockwise along the prox- 

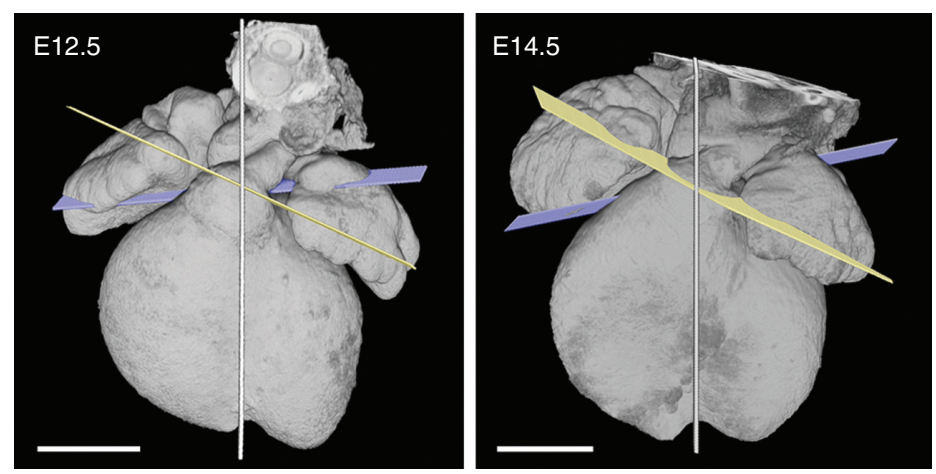

Figure 4. Comparison of arterial valve planes between E12.5 and E14.5. Both the relative orientation of the valve planes, and their location with respect to the midseptal plane (shown), remain unchanged between E12.5 and E14.5. Scale bars, $0.5 \mathrm{~mm}$.

imal to distal axis of the outflow tract. One cushion extends from a noticeably broad, but thin, base attached to the inner surface of the right ventricular wall. It twists to occupy a superior position in the central region of the outflow tract. Finally, at its most distal end, it occupies the left side of the outflow tract. A second, initially more bulbous, cushion begins from the left side of the outflow tract as it emerges from the right ventricle, being attached to the right side of

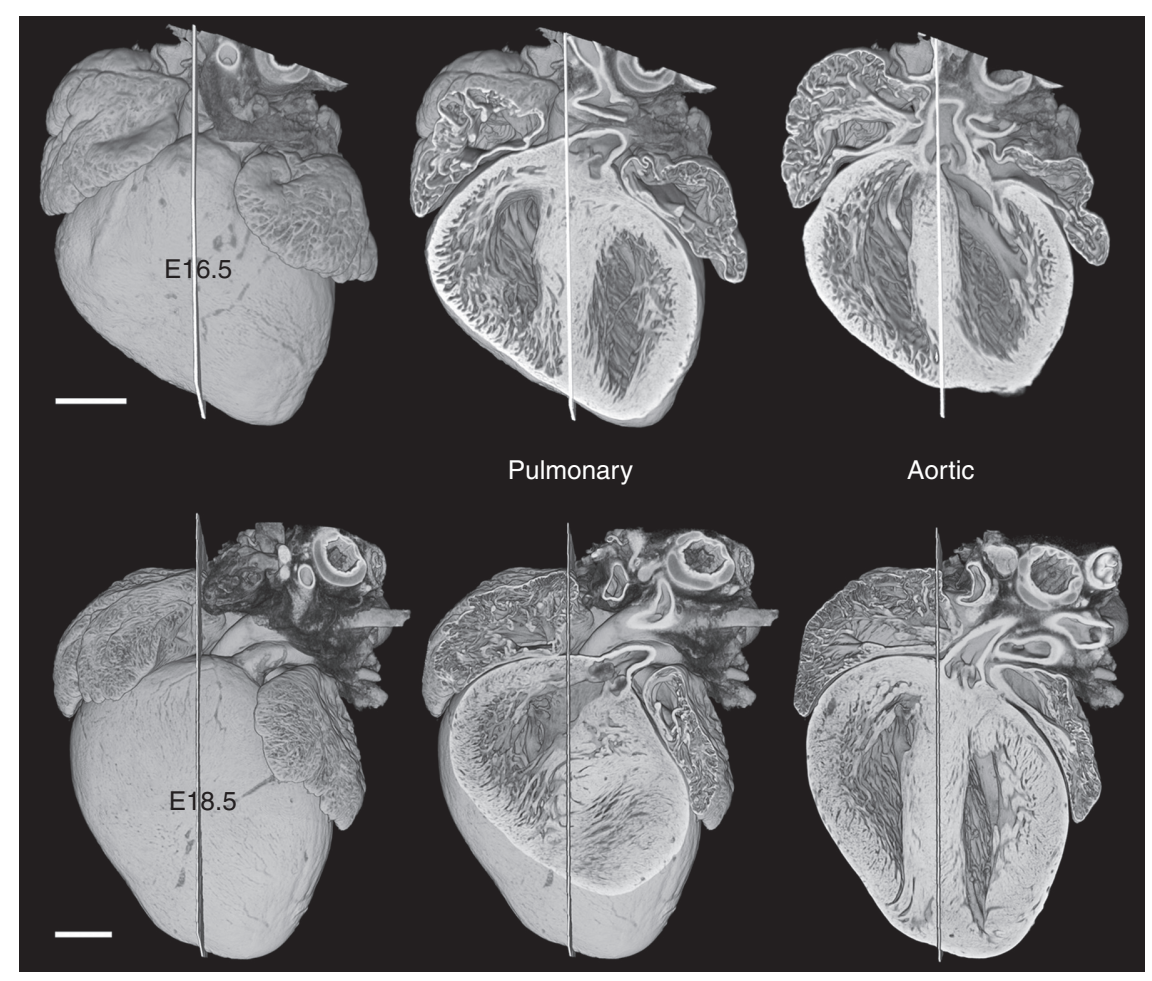

Figure 5. By E16.5, the entrance to the pulmonary root lies clearly to the left of a midseptal plane, shifting further to the left by full term (E18.5). Over the same period, the aortic valve also shifts leftward, straddling the left face of the interventricular septum by E18.5. Scale bars, $0.5 \mathrm{~mm}$. 
the developing muscular ventricular septum. More distally, this cushion lies on the inferior side of the central outflow, and then twists to the right side at its most distal end (Fig. 6).

As a result of this spiraling arrangement, the two cushions have been variously termed left/ superior/parietal and right/inferior/septal, respectively. Over the course of the next day (E11.5-E12.5), two additional cushions appear in the central portion of the outflow tract. Lying on either side between the main cushions, these "intercalated" cushions will contribute to forming the arterial valvar primordia. By tracking cushion arrangement in this region, it is clear that at least the central portion of the outflow tract undergoes further progressive rotation as the more distal outflow separates into distinct vessels. As a result, the intercalated cushions shift from a largely left-to-right to a more ventrodorsal orientation (Fig. 7).
Spiraling and rotation along the axis of the outflow tract has a crucial consequence for subsequent development. As a result of this arrangement of the cushions, the entrance to the future pulmonary channel will lie at the superior edge of the right ventricular chamber, whereas that of the aortic channel will be much more central (Fig. 7).

\section{SEPARATION OF THE PULMONARY AND AORTIC CHANNELS}

Fusion of the apposing surfaces of the parietal and septal outflow cushions along the length of the outflow tract separates the pulmonary and aortic channels, each of which becomes progressively enclosed within its own walls. This transformation begins in the distal outflow and progresses ever more proximally. At their most proximal ends, fusion of the parietal and septal

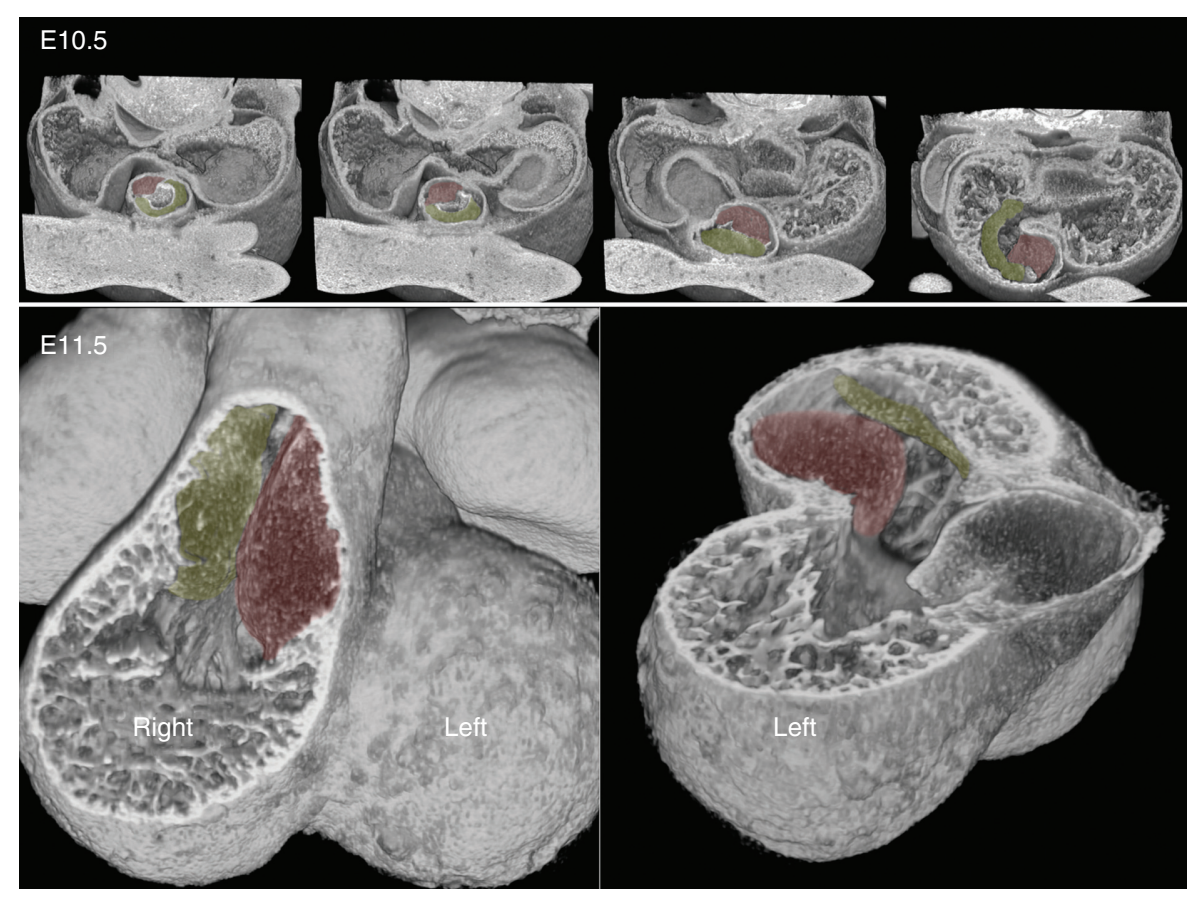

Figure 6. At E10.5, progressive transverse erosion of the outflow tract from its distal portion toward the heart reveals the spiral arrangement and differing shapes of the septal (red) and parietal (green) outflow tract cushions. At E11.5, erosions reveal how the proximal portion of the two cushions face each other at the entrance to the outflow tract. Note how the base of the more bulbous septal cushion attaches to the right face of the growing interventricular septum. 

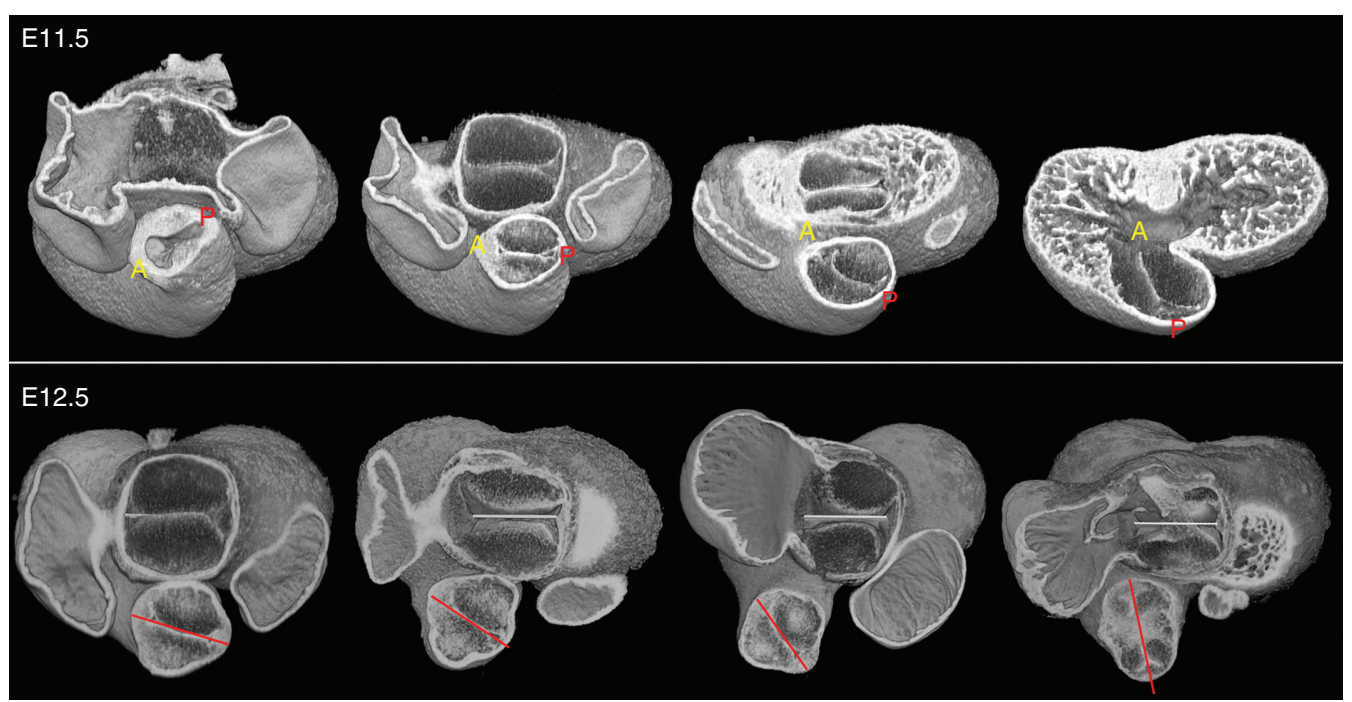

Figure 7. At E11.5, the spiral arrangement of the aortic $(\mathrm{A})$ and pulmonary $(\mathrm{P})$ arterial channels within the single outflow tract is revealed by progressive transverse erosion planes. Further rotation occurs during E12.5. This can be seen by eroding successive developmental stages at a fixed transverse plane at approximately midlevel through the outflow tract. This region contains the intercalated cushions. The erosion plane reveals the trifoliate appearance of the valve primordia in the oldest sample. Models are oriented with respect to the plane of apposition (marked) between the superior and inferior cushions of the atrioventricular (AV) junction.

cushions is a critical step toward separation of left and right ventricles. As the faces of the cushions merge together in this region, they form a thick "hanging wall," or shelf, of cushion tissue. This forms a physical barrier separating the entrances to the pulmonary and aortic channels (Fig. 8).

Furthermore, as a result of cushion spiraling, the entrances to the aortic and pulmonary channels lie on significantly divergent axes. That of the pulmonary channel is approximately aligned with the overall axis of the outflow tract, passing leftward as it ascends. In contrast, the entrance to the aortic channel is relatively offset, passing rightward as it enters the base of the outflow tract. This places it in a more appropriate orientation for draining from the left side of the heart, although it remains at this stage above the right ventricle (Fig. 9).

By E12.5 then, the arrangement and subsequent fusion of the outflow tract cushions has not only enabled separation of the aortic and pulmonary vessels, it has also placed their entrances in orientations that presage their ulti- mate chamber-specific function. Cushion fusion has also created a physical partition that restricts the drainage offered by each channel to distinct regions of the right ventricle.

\section{CLOSURE OF THE INTERVENTRICULAR COMMUNICATION}

Throughout this period, access to the aortic root from the left ventricular cavity is only possible because of persistence of an interventricular communication across the crest of the muscular ventricular septum (Fig. 10A).

Complete separation of the left and right ventricles therefore requires a mechanism for closing the interventricular communication, while simultaneously establishing unfettered passage from the left ventricle to the aortic root. Once again, a key role is played by outflow cushion tissue. As we have seen (Fig. 6) from its first appearance, the septal cushion of the outflow tract extends deep into the future right ventricle, attaching to the right side of the developing ventricular septum. By early E12.5, the base 


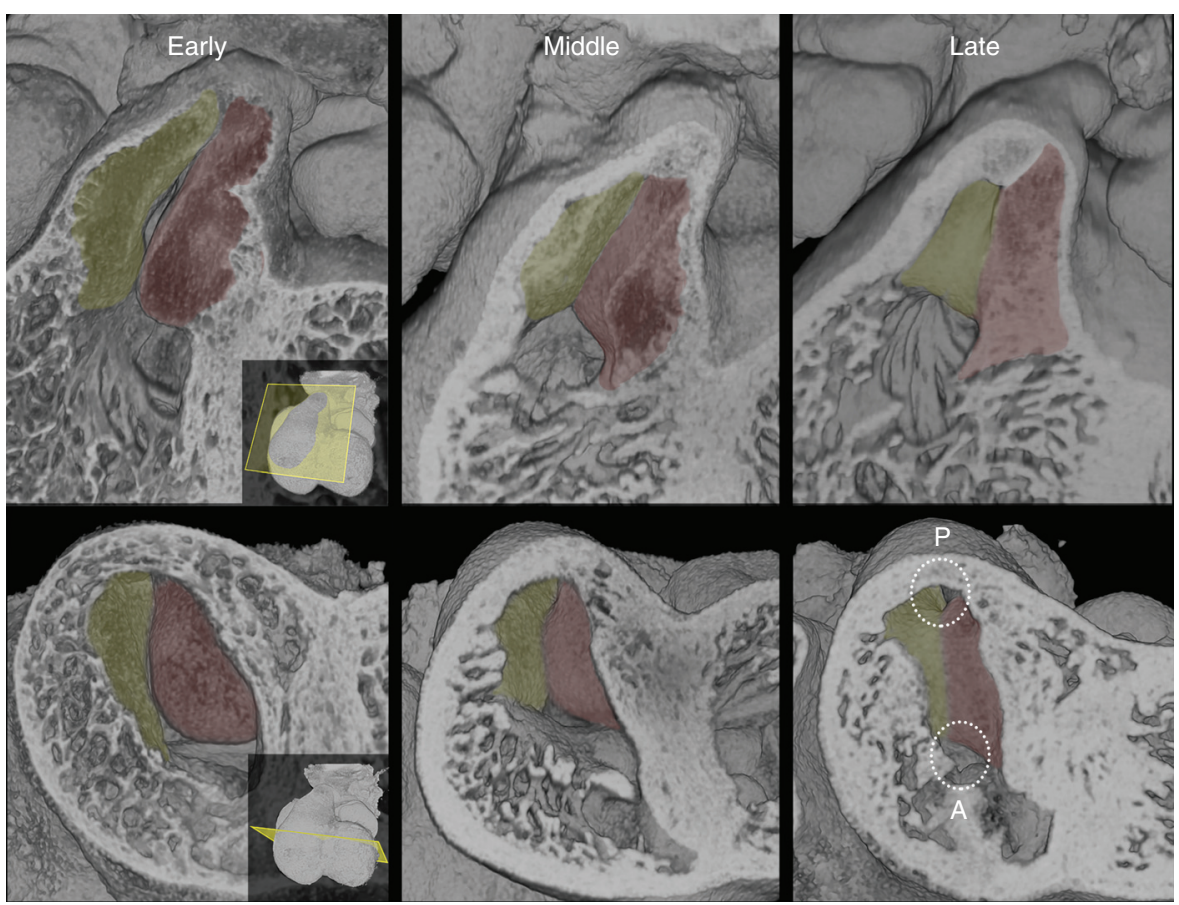

Figure 8. Fusion of the proximal outflow cushions during E12.5. Apposing faces of the septal (red) and parietal (green) cushions fuse during E12.5, creating a shelf of tissue that separates the entrances to the pulmonary (P) and aortic (A) roots. Cushion fusion is visualized through erosion of three-dimensional (3D) models in either fourchamber (top row) or axial (bottom row) planes.

of this cushion is a bulbous structure covering the cranial half of the septum. Because of this, communication between the left and right ventricles is restricted to the caudal half of the ventricular septal crest, a region that lies directly beneath the aortic root (Fig. 10B).

Closure of this remaining interventricular communication is now achieved by a sequence of tissue fusions involving the bulbous end of the septal cushion. As fusion between this and the adjacent proximal portion of the parietal cushion establishes a growing barrier of tissue separating the pulmonary and aortic root entrances, the septal cushion also begins to fuse along an orthogonal axis. Merging of its convex face with the adjacent superior atrioventricular cushion closes most of the gap that allows communication across the caudal region of the ventricular septal crest (Fig. 11).

In doing so, this effectively walls in the base of the aortic root, enabling it to traverse the ventricular septum to the left ventricle. From a topological point of view, the original interventricular space directly above the crest of the sep-

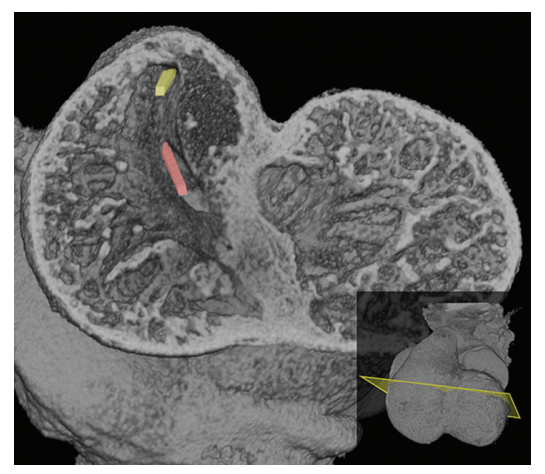

Figure 9. Midventricular erosion plane at E11.5 viewed from the apical side. The spiral arrangement of outflow tract cushions results in divergent orientations for the entrances to the pulmonary (yellow) and aortic (red) channels. 


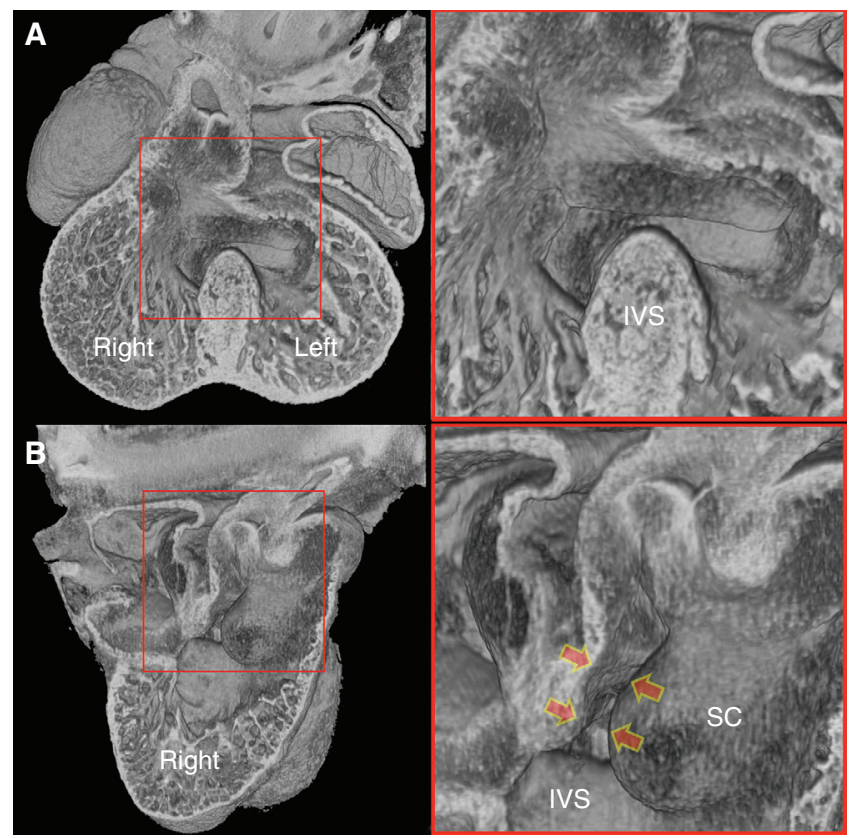

Figure 10. E12.5 heart. (A) Four-chamber erosion plane. After separation of the aortic and pulmonary vessels, the aortic root continues to lie above the right ventricle. The interventricular communication across the crest of the interventricular septum (IVS) permits access to the aortic root from the left ventricle. (B) Erosion through the right ventricle. The bulbous, septal cushion (SC) occludes much of the interventricular communication, restricting it to the most caudal region. Fusion with the adjacent cushion tissue completes sealed access of the aortic root to the left ventricle (arrows).

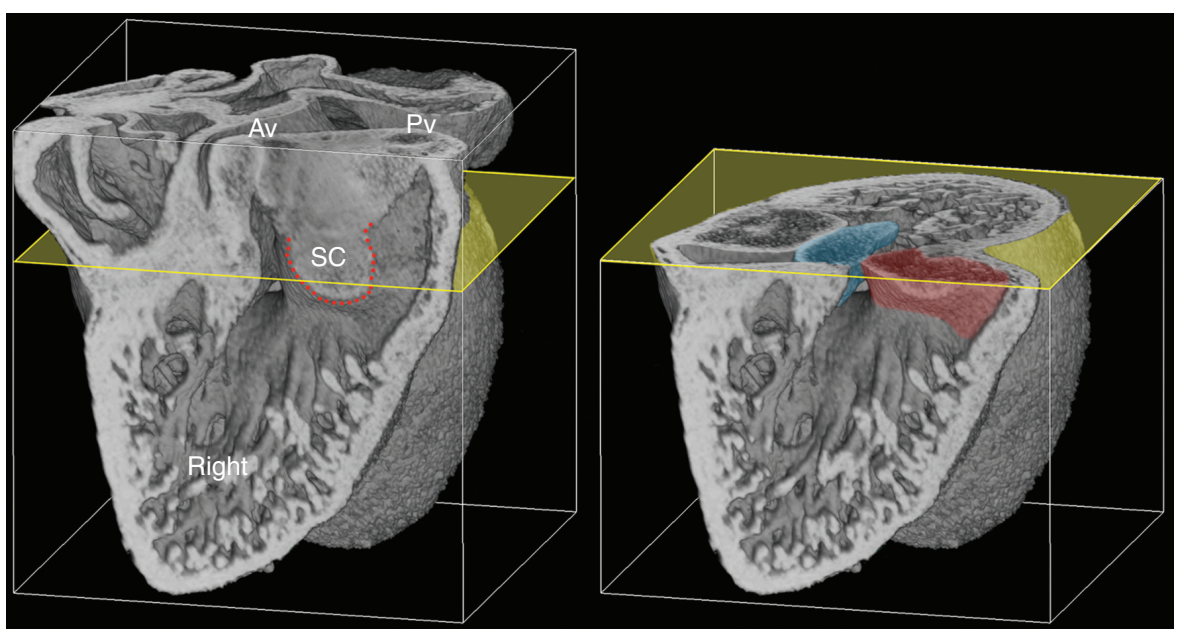

Figure 11. E12.5 heart, eroded from the right. The erosion plane reveals the extent of fusion between the proximal septal (SC) and parietal cushions (red dotted line) and the resulting separation of openings to the aortic (Av) and pulmonary $(\mathrm{Pv})$ valves. Further axial erosion (yellow) shows that unique access of the aortic root to the left ventricle is achieved by fusion between the SC (red) and the superior atrioventricular cushion (blue). Note the adjacent residual interventricular communication. 
tum is not itself closed by this process. Rather its caudal half is converted into the entrance to the outflow tract serving the left ventricle.

By E13.5, the only remnant of interventricular communication lies at the most caudal end of the interventricular septal crest. Final closure is completed by the so-called "tubercles." These are protuberances from the right side of the atrioventricular cushions that fuse with the rightmost edge of the caudal interventricular septum (IVS). Fusion seals the remaining passage between left and right ventricles, which now each possess separate arterial drainage (Fig. 12).

\section{CONCLUSIONS}

Generations of cardiac anatomists have highlighted both the beauty of the mammalian heart and the intricacy of the steps that transform it from pulsatile tube into a mature, four-chambered organ. With modern imaging methods, we can now all share an appreciation of these events, using digital 3D models to retrace their steps and clarify the most stubborn and intrac- table problems of embryonic heart morphology. Here we have reexamined how, subsequent to expansion of the atrioventricular canal, partition of the ventricular loop into left and right ventricles results in separate chambers, each with their own arterial root. With the advantage of $3 \mathrm{D}$ models, it is clear that both arterial roots retain their location over the right ventricular chamber throughout growth of the muscular ventricular septum. The only major shift is the reorientation of the axis of the aortic root. This change only becomes possible when division of aortic and pulmonary roots extends through the central part of the outflow tract, separating their respective valves.

Subsequent closure of the communication between the two ventricles, and the establishment of left ventricular drainage through the aortic root, requires a sequence of events in which the outflow tract cushions play a central role. The spiral arrangement of parietal and septal cushions places the entrances of the pulmonary and aortic channels over distinct regions of the right ventricle. This separation is then consolidated by fusion of the proximal cushion re-

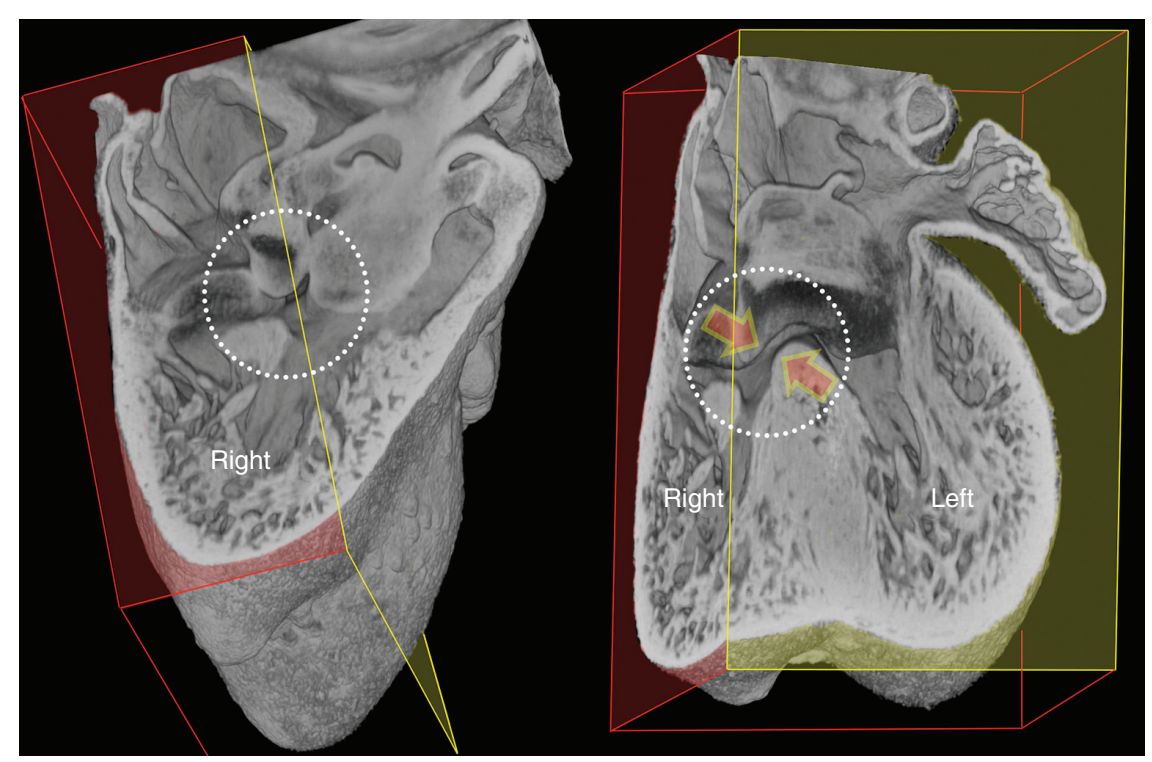

Figure 12. E13.5 heart, eroded from the right (red) and in the four-chamber plane (yellow). The remaining interventricular communication (circled) is closed by fusion (red arrows) between the "tubercles" (protuberances of the right margins of the atrioventricular cushions) and the adjacent right face at the caudalmost crest of the interventricular septum. 
gions, creating a shelf of tissue between the entrances to the two roots. Fusion of the bulbous base of the septal cushion with the adjacent atrioventricular cushion closes much of the remaining left-to-right ventricular communication. At the same time, it creates a tunnel from the aortic root that crosses the caudal crest of the muscular ventricular septum. Final closure is then achieved by fusion of the tubercles of both atrioventricular cushions with the right side of the septum. (This results in the asymmetric and rightward tilt in the septum that is so evident in more caudal four-chamber views of the mature embryonic heart.)

This morphological account of course only provides a skeleton for our understanding of these events and is derived only from studies of the mouse heart. Far fewer equivalent datasets are available for human heart development, but our account is, to our knowledge, largely consistent with these and with earlier interpretations of histological studies. Changes in cellular composition, muscularization, and the distribution of different cell lineages all contribute to functional differentiation of the heart. The challenge, therefore, is to integrate them into a comprehensive account. Even the partial view we have presented, nevertheless, suggests some important conclusions. The right-sided location of both entrances to the arterial roots, and the shift in orientation of the aortic root, are surely of significance for understanding the etiologies of abnormalities involving the positioning of the great vessels. Similarly, the central role played by the major cushions in both outflow and ventricular septation accounts for the frequent association of abnormalities in both chamber drainage and separation. Finally, it is clear that closure of interventricular communication is not simply the result of growth of a septum to occlude the space above its crest. Rather it is achieved via changes involving several component tissues and steps. As a consequence, ventricular septal defects could have multiple distinct etiologies. These could include abnormalities in the positioning or growth of either the outflow cushions or the interventricular septal wall. They could also result from lesions in any of the fusions that our 3D models have illustrated normally occur between these tissues.

\section{ACKNOWLEDGMENTS}

We thank our colleagues first at the MRC National Institute for Medical Research and more recently at the Francis Crick Institute for supporting development of HREM imaging and provision of the many mouse embryos that provided the basis for our studies. T.J.M. was supported by the Francis Crick Institute, which receives its core funding from Cancer Research UK (FC001157, FC001117), the UK Medical Research Council (FC001157, FC001117), and the Wellcome Trust (FC001157, FC001117). T.J.M. was also supported by the Medical Research Council (U117562103). 


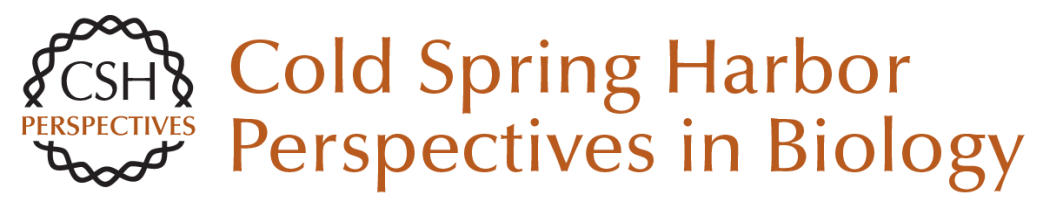

\title{
3D Anatomy of the Developing Heart: Understanding Ventricular Septation
}

\author{
Timothy J. Mohun and Robert H. Anderson
}

Cold Spring Harb Perspect Biol 2020; doi: 10.1101/cshperspect.a037465 originally published online January 27, 2020

\section{Subject Collection Heart Development and Disease}

Epicardium in Heart Development

Yingxi Cao, Sierra Duca and Jingli Cao

Cardiac Neural Crest

Hiroyuki Yamagishi

The Endocardium and Heart Valves Bailey Dye and Joy Lincoln

Long Noncoding RNAs in Cardiac Development Michael Alexanian and Samir Ounzain

Genetic Basis of Human Congenital Heart Disease Shannon N. Nees and Wendy K. Chung

Cardiopharyngeal Progenitor Specification: Multiple Roads to the Heart and Head Muscles Benjamin Swedlund and Fabienne Lescroart

Genetic and Epigenetic Control of Heart Development Brynn N. Akerberg and William T. Pu

Formation and Growth of Cardiac Lymphatics during Embryonic Development, Heart Regeneration, and Disease

Dana Gancz, Gal Perlmoter and Karina Yaniv
In Vivo and In Vitro Genetic Models of Congenital

Heart Disease Uddalak Majumdar, Jun Yasuhara and Vidu Garg

Development of the Cardiac Conduction System

Samadrita Bhattacharyya and Nikhil V. Munshi

3D Anatomy of the Developing Heart:

Understanding Ventricular Septation

Timothy J. Mohun and Robert H. Anderson

Cardiac Morphogenesis: Specification of the

Four-Chambered Heart

Vincent Christoffels and Bjarke Jensen

The Chicken as a Model Organism to Study Heart

Development

Johannes G. Wittig and Andrea Münsterberg

The First Heartbeat--Origin of Cardiac Contractile

Activity

Richard C.V. Tyser and Shankar Srinivas

Xenopus: Experimental Access to Cardiovascular

Development, Regeneration Discovery, and

Cardiovascular Heart-Defect Modeling

Stefan Hoppler and Frank L. Conlon

Reptiles as a Model System to Study Heart

Development

Bjarke Jensen and Vincent M. Christoffels

For additional articles in this collection, see http://cshperspectives.cshlp.org/cgi/collection/

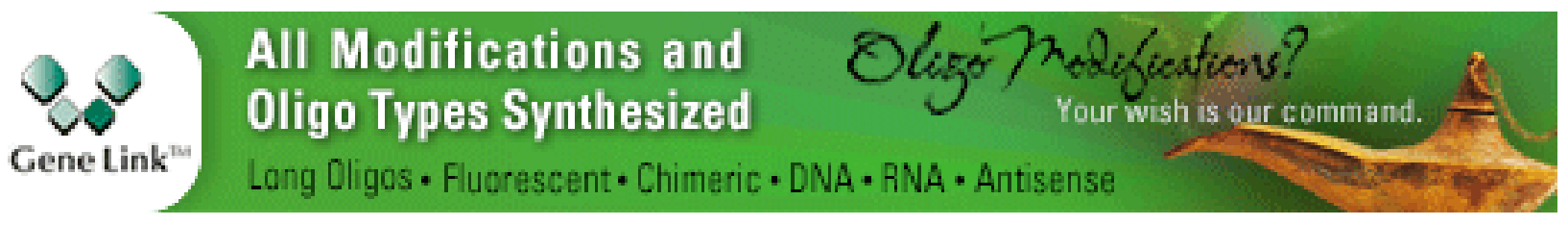

Copyright @ 2020 Cold Spring Harbor Laboratory Press; all rights reserved 